\title{
CONTINUOUS CHARACTERS OF COMPACT SEMIGROUPS
}

\author{
BY \\ RONALD O. FULP( $\left.{ }^{1}\right)$
}

Introduction. It is well known that each continuous character of each closed subgroup of a locally compact commutative group $G$ can be extended to a continuous character of $G$ (see [3]). It is equally well known that the corresponding statement fails to be true about locally compact commutative semigroups. The statement is false even for discrete semigroups (see, for example, [2] or [4]). Several papers have been written concerning the problem as to when it is possible to extend characters defined on subsemigroups of discrete commutative semigroups. The object of this paper is to carry out a similar investigation for compact commutative semigroups. More precisely, one question which is investigated here is as follows: when is it possible to extend a continuous character of a closed subsemigroup of a compact commutative semigroup $S$ to all of $S$ ? The problem still lacks precision due to the fact that there are several ways of defining a character of a semigroup. We consider two of the usual definitions in this paper and obtain solutions of the problem in each case. These results run parallel to the work of Fulp [2], Hill [4], and Ross [6] on extending characters of discrete semigroups.

Incidental to the main investigation, we obtain various other theorems along the way. For example, we obtain some results which bear on the question as to when the semigroup of characters of a compact commutative semigroup is isomorphic to the semigroup of characters of some one of its closed subsemigroups. We also show that the semigroup of idempotent characters of a compact commutative semigroup is always isomorphic to the semigroup of idempotent characters of some compact totally disconnected commutative semigroup.

Throughout the paper we abbreviate Hausdorff topological semigroup to simply semigroup. If $S$ is any semigroup, $E(S)$ will denote the set of idempotents of $S$. If $S$ is commutative, $E(S)$ is a subsemigroup of $S$ and, in any case, there is a natural partial order $\leqq$ on $E(S)$ defined by $e \leqq f$ if and only if $e f=e=f e$.

The multiplicative group of complex numbers $z$ such that $|z|=1$ will be denoted by $\Omega$. The topological group $\Omega$ with an isolated zero adjoined will be denoted by $\Omega^{0}$. If $T$ is a semigroup, then to say that $\chi$ is a character of $T$ means that $\chi$ is a homomorphism from $T$ into $\Omega^{0}$. If $T$ is commutative, then the set $X$ of all continuous characters of $T$ may be given a semigroup structure by endowing it with the

Presented to the Society, April 22, 1967; received by the editors March 2, 1967 and, in revised form, February 15, 1968.

(1) The author of this research was supported in part by NASA Grant NGR 44-005-037. 
compact-open topology and by defining the product of two characters as the usual pointwise product of two functions. The resulting semigroup will be denoted by $T^{\wedge}$. The group of units of $T^{\wedge}$ will be denoted by $T^{*}$ and its members will be called unit-characters of $T$. It is known (see, for example, Austin [1]) that if $T$ is compact and commutative, then $T^{\wedge}$ and $T^{*}$ are discrete.

Finally, if $\chi$ is a character of a semigroup $T$, then the support of $\chi$, denoted by $S_{x}$, is defined to be that subsemigroup of $T$ consisting of those $x \in T$ such that $\chi(x) \neq 0$. Note that $\chi$ is a unit-character of $T$ if and only if $S_{x}=T$.

1. Unit-characters. In this section we consider the problem of extending continuous unit-characters defined on closed subsemigroups of compact commutative semigroups. Essentially this is accomplished by showing that a continuous unit-character of a compact commutative semigroup $S$ is determined by its values on the minimal ideal of $S$. Since the minimal ideal of $S$ is a compact topological group we are able (modulo some translations of subgroups of a semigroup) to reduce the problem to the theory of topological groups where a solution is already available.

The first theorem of this section, while not needed in the subsequent development, provided the motivation for most of this section of the paper. Our original idea was to "factor" the unit-characters of $S$ through the maximal group homomorphic image of $S$. Our first theorem shows that the maximal group homomorphic image of $S$ is the same as the minimal ideal of $S$.

LEMMA 1.1. Assume $S$ is a compact commutative semigroup. Then the following statements are equivalent:

(1) $\rho$ is the maximal cancellative congruence of $S$,

(2) $\rho$ is the maximal group congruence of $S$,

(3) $(x, y) \in \rho$ if and only if $x a=y a$ for some $a \in S$, and

(4) $(x, y) \in \rho$ if and only if $x e=y e$ where $e$ is the least element of $E(S)$.

Proof. That (1) and (3) are equivalent is well known. That (1) and (2) are equivalent follows from the fact that each compact commutative cancellative semigroup is a topological group (see Hewitt and Ross [3]). It is clear that (4) implies (3). To see that (3) implies (4), assume $x$ and $y$ are members of $S$ such that $x a=y a$ for some $a \in S$. Since $S$ is compact, $E(S)$ has a least element $e$ with respect to the natural partial order on $E(S)$ (see Schwarz [7]). Moreover, ae belongs to the maximal subgroup of $S$ which contains $e$. Thus there is a net $j$ of natural numbers such that the net $a^{j}$ defined by $\alpha \rightarrow(a e)^{j(\alpha)}$ has limit $e$. The equation $x a=y a$ implies that $x(a e)^{j(\alpha)}=y(a e)^{j(\alpha)}$ for each $\alpha$. Thus $x e=y e$ and (4) follows.

THEOREM 1.2. Suppose $S$ is a compact commutative semigroup, that $e$ is the least element of $E(S)$, and that $C$ is the maximal cancellative homomorphic image of $S$. Then

1. $C$ is a topological group and is the maximal group homomorphic image of $S$, 
2. $C$ is iseomorphic to the maximal subgroup of $S$ which contains $e$, and

3. the function $\psi$ defined by $x \rightarrow x e$ is a continuous homomorphism from $S$ onto the maximal subgroup of $S$ which contains $e$ (thus $\psi$ may be viewed as the canonical homomorphism from $S$ onto its maximal cancellative homomorphic image).

Proof. Statement 3 is evident modulo the fact that $S e$ is the maximal subgroup of $S$ containing $e$ (see Hofmann and Mostert [5]). Statement 1 is an immediate consequence of the lemma.

We now prove 2 of the theorem. Let $\psi$ denote the map from $S$ onto $S e$ defined by $x \rightarrow x e$. Let $\rho$ denote the relation on $S$ defined by $(x, y) \in \rho$ if and only if $x a=y a$ for some $a \in S$. Lemma 1.1 implies that $(x, y)$ belongs to $\rho$ if and only if $\psi(x)=\psi(y)$. It follows from the fundamental homomorphism theorem that $S e$ and $S / \rho$ are iseomorphic. But $S / \rho$ is clearly the maximal cancellative homomorphic image of $S$. The theorem follows.

THEOREM 1.3. Assume $S$ is a compact commutative semigroup, that $e$ is the least element of $E(S)$, and that $H$ is a closed subsemigroup of $S$. If $\chi$ is a continuous unitcharacter of $H$, then the following statements are equivalent:

1. there is an extension of $\chi$ which is a continuous unit-character of $S$,

2. $(x, y, a) \in H \times H \times S$ and $x a=y a$ imply $\chi(x)=\chi(y)$, and

3. $(x, y) \in H \times H$ and $x e=y e$ imply $\chi(x)=\chi(y)$.

Proof. That 2 and 3 are equivalent follows from Lemma 1.1. It is evident that 1 implies each of 2 and 3 . We show that 3 implies 1 . Let $\psi$ denote the function from $S$ onto $S e$ defined by $x \rightarrow x e$. Consider the diagram:

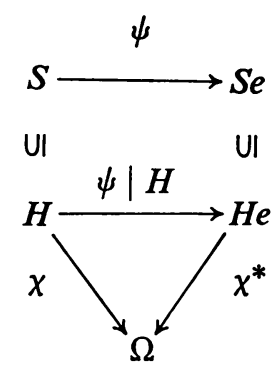

where $\chi^{*}$ is the function from $H e$ into $\Omega$ defined by $h e \rightarrow \chi(h)$ for $h \in H$. It is evident that $\chi^{*}$ is well defined and that $\chi^{*}$ is a homomorphism which makes the above diagram commutative. That $\chi^{*}$ is continuous follows immediately from the fact that if $F$ is a closed subset of $\Omega$, then $\chi^{-1}(F)$ is a compact subset of $H$ and $\chi^{*-1}(F)=\psi\left(\chi^{-1}(F)\right)$ is a closed subset of $H e$. Since $S$ and $H$ are compact, so are $S e$ and $\mathrm{He}$. It follows that $\mathrm{Se}$ and $\mathrm{He}$ are topological groups. Let $\chi^{\prime}$ denote a (group) character of $S e$ which is continuous and which extends $\chi^{*}$. Clearly $\chi^{\prime 0} \psi$ is a continuous unit character of $S$ which extends $\chi$.

Corollary 1.4. Assume $S$ is a compact commutative semigroup and that $G$ is a closed subsemigroup of $S$. The following statements are equivalent: 
1. each continuous unit character of $H$ can be extended to a continuous unitcharacter of $S$, and

2. if $(x, y, a) \in H \times H \times S$ and $x a=y a$, then $x e=y e$ where $e$ is the least element of $E(H)$.

Proof. Assume 2 is true, that $\chi$ is a continuous unit-character of $H$, and that $(x, y, a) \in H \times H \times S$ such that $x a=y a$. It then follows that $x e=y e$ where $e$ is the least element of $E(H)$. Thus $\chi(x)=\chi(x e)=\chi(y e)=\chi(y)$ and by Theorem 1.3, $\chi$ can be extended to $S$.

Now assume 1 is true and that $x$ and $y$ are members of $H$ such that $x e \neq y e$, but $x a=y a$ for some $a \in S$. Note that $x e$ and $y e$ are in the same maximal subgroup $G$ of $H$ and that $G$ is precisely the maximal subgroup containing $e$. Let $\chi$ denote any continuous (group) character of $G$ such that $\chi(x e) \neq \chi(y e)$. Clearly the function $\chi^{\prime}$ defined by $h \rightarrow \chi(h e)$ for $h \in H$ is a continuous unit character of $H$. Let $\chi^{*}$ denote a continuous unit-character of $S$ which extends $\chi^{\prime}$. Since $x a=y a, \chi^{*}(x)=\chi^{*}(y)$ and $\chi(x e)=\chi^{*}(x)=\chi^{*}(y)=\chi(y e)$ contrary to the choice of $\chi$. The corollary follows.

REMARK. If $S$ is a compact commutative semigroup and $x$ is in $S$, then there is one and only one idempotent $e$ of $S$ which belongs to the closure of the subsemigroup of $S$ generated by $x$. The idempotent $e$ will be called the idempotent belonging to $x$ and will be denoted by $e_{x}$.

COROLLARY 1.5. If $S$ is a compact commutative semigroup, then each continuous unit-character of each closed subsemigroup of $S$ has an extension which is a continuous unit-character of $S$ if and only if $(x, y, a) \in S \times S \times S$ and $x a=y a$ imply $x e_{x} e_{y}=y e_{x} e_{y}$.

The proof of Corollary 1.5 involves details similar to those of the proof of Corollary 1.4 and is omitted.

We now seek conditions under which a continuous unit-character of a closed subsemigroup has a unique extension. For compact commutative semigroups, the following theorem reduces the question to the corresponding question about continuous group characters of topological groups.

THEOREM 1.6. Suppose $S$ is a compact commutative semigroup, that $e$ is the least idempotent of $S$, and that $\chi$ is a continuous unit-character of some closed subsemigroup $H$ of $S$. Then any two extensions of $\chi$ to a continuous unit-character of $S$ agree on He. For each $\chi$ which has an extension to $S$, let $\chi_{e}$ denote the restriction of any such extension to He. Then $\chi$ has a unique extension to $S$, if and only if the continuous group character $\chi_{e}$ of the subgroup He of the topological group Se has a unique extension to $\mathrm{Se}$.

Proof. It is clear that any two continuous unit-characters of $S$ which agree on $\mathrm{H}$ also agree on $\mathrm{He}$. The first statement of the conclusion of the theorem follows immediately. The remainder of the theorem follows from the fact that any continuous unit-character of $S$ is determined by its values on $S e$. 
Remark. Assume $S$ is a compact commutative semigroup and that $H$ is any closed subsemigroup of $S$. Let $\Phi$ denote the function from $S^{*}$ into $H^{*}$ defined by $\Phi(\chi)=\chi \mid H$. Theorem 1.3 asserts that $\Phi$ is onto if and only if $(x, y, a) \in H \times H \times S$ and $x a=y a$ imply $x e=y e$ where $e$ is the least element of $E(H)$. Theorems 1.3 and 1.6 imply that $\Phi$ is an iseomorphism if and only if

1. $(x, y, a) \in H \times H \times S$ and $x a=y a$ imply $x e=y e$ (as above $e$ is the least element of $E(H))$, and

2. each two continuous group characters of the topological group $S e$ which agree on the subgroup $\mathrm{He}$ of $\mathrm{Se}$ are identical.

Another theorem along these lines is

THEOREM 1.7. Assume $S$ is a compact commutative semigroup, that $H$ is a closed subsemigroup of $S$, that $e$ and $f$ are the least elements of $E(S)$ and $E(H)$ respectively, and that $\Gamma$ is the function from $(H e)^{*}$ into $H^{*}$ defined by $\Gamma(\chi)(h)=\chi(h e)$ for each $\chi \in(H e)^{*}$ and $h \in H$. Then

1. $\Gamma$ is always a bicontinuous monomorphism and is an iseomorphism if and only if $h_{1}, h_{2}$ in $H$ and $h_{1} e=h_{2}$ e imply $h_{1} f=h_{2} f$,

2. $S^{*}$ and $(\mathrm{Se})^{*}$ are iseomorphic, and

3. $S^{*}$ and $H^{*}$ are iseomorphic if and only if the topological groups Se and Hf are iseomorphic.

Proof. The proof of 1 is straightforward. Statement 2 is a consequence of 1 , while 3 follows from 2 and the Pontryagin-Van Kampen duality theorem (see Hewitt and Ross [3, p. 378]).

REMARK. Statement 2 of the theorem is due to Schwarz [7]. One consequence of the theorem is the following: if $S$ and $T$ are compact commutative semigroups and $e$ and $f$ are the least elements of $E(S)$ and $E(T)$ respectively, then $S^{*}$ is iseomorphic to $T^{*}$ if and only if the topological groups $S e$ and $T f$ are iseomorphic.

2. Characters. We now turn our attention to continuous characters of compact commutative semigroups. First we need further notation. For each $e \in E(S)$, the set $\left\{x \in S \mid e_{x}=e\right\}$ is a subsemigroup of $S$ which we shall denote by $P_{e}$. For $e$ and $e^{\prime}$ in $E(S), P_{e} P_{e^{\prime}} \subseteq P_{c e^{\prime}}$. Moreover, the maximal subgroup of $S$ which contains $e \in E(S)$ is precisely $e P_{e}$. This group will be denoted by $G_{e}$. The set $P_{e}$ may be neither open nor closed but $G_{e}$ is always closed. These results are due to Schwarz (see [7] or [8]) and others. Finally, $e \in E(S)$ is said to be a generating idempotent of $S$ if and only if the open prime ideal $\bigcup\left\{P_{f} \mid e \pm f\right\}$ of $S$ is also closed. If $F$ is a subset of some subsemigroup $H$ of $S$ and if $e \in E(S)$, then we say that $e$ induces $F$ if and only if $F=H \cap \bigcup\left\{P_{f} \mid e \leqq f\right\}$.

THEOREM 2.1. Assume $S$ is a compact commutative semigroup, that $\chi$ is a continuous character of a closed subsemigroup $H$ of $S$, and that $e$ is the least element of $E\left(S_{\chi}\right)$. In order that $\chi$ admit an extension which is a continuous character of $S$, it is necessary and sufficient that either $\chi$ is identically zero or 
1. there exists a generating idempotent $f$ of $S$ which lies under e and which has the property that if $x$ is a member of the maximal subgroup of $H$ containing $e$ such that $x f=f$, then $\chi(x)=1$, and

2. there exists a generating idempotent $f^{\prime}$ of $S$ which induces $S_{\chi}$.

Proof. The theorem is evident if either $S_{x}=S$ or $S_{x}$ is void. We assume that neither of these is the case. Observe that in order to extend $\chi$ to $S$, it follows from Theorem 1.3 that it is necessary and sufficient that there exists an open and closed prime ideal $P$ of $S$ such that

(1) $(S \backslash P) \cap H=S_{x}$, and

(2) $(x, y, a) \in S_{x} \times S_{x} \times(S \backslash P)$ and $x a=y a$ imply $\chi(x)=\chi(y)$.

If $P$ is an open and closed prime ideal satisfying (1) and (2), let $f$ denote the least element of $E(S \backslash P)$. The idempotent $f$ then satisfies 1 and 2 of the theorem.

Suppose, on the other hand, that $f$ satisfies 1 and that $f^{\prime}$ satisfies 2 of the theorem. If $Q=\bigcup\left\{P_{g} \mid f \leqq g\right\}$ and $Q^{\prime}=\bigcup\left\{P_{g} \mid f^{\prime} \leqq g\right\}$, then $e \in Q \cap Q^{\prime}$ and thus $Q \cap Q^{\prime}$ is a nonvoid open and closed subset of $S$ whose complement in $S$ is a nonvoid open and closed prime ideal $P$ of $S$. The open and closed prime ideal $P$ satisfies (1) and (2) above (to see that (2) is satisfied observe that $x a=y a$ implies that $(x e)(y e)^{-1} f=f$ and apply 1).

In all that follows, $S$ denotes a compact commutative semigroup, $H$ denotes a closed subsemigroup of $S$, and $F$ a nonvoid subset of $H$ whose complement in $H$ is a proper open and closed prime ideal of $H$. Let $e$ denote the least element of $E(F)$, $H_{e}$ the maximal subgroup of $H$ containing $e$, and $E_{e}$ the set of all generating idempotents of $S$ lying below $e$ (possibly $e \in E_{e}$ ). Note that $E_{e}$ is directed upward and that actually each two elements of $E_{e}$ have a least upper bound in $E_{e}$. If $\left(f, f^{\prime}\right)$ is an ordered pair in $E_{e} \times E_{e}$ such that $f \leqq f^{\prime}$, let $\varphi_{f f^{\prime}}$ denote the function from the group $\left(f H_{e}\right)^{*}$ into the group $\left(f^{\prime} H_{e}\right)^{*}$ defined by $\varphi_{f f^{\prime}}(\chi)\left(x f^{\prime}\right)=\chi(x f)$ for each $x \in H_{e}$ and $\chi \in\left(f H_{e}\right)^{*}$. It is easy to verify that $\varphi_{f f^{\prime}}$ is well defined and that it is a homomorphism. The system

$$
\left[\left\{\left(f H_{e}\right)^{*}\right\}_{f \in E_{e}} ;\left\{\varphi_{f f^{\prime}}\right\}_{f \leqq f^{\prime}}\right]
$$

is a direct system of groups whose direct limit we denote by $H_{e}^{* \infty}$. Let $\psi$ denote the function from $H_{e}^{* \infty}$ into $H_{e}^{*}$ defined by $\psi(t)(x)=\chi(x f)$ where $x \in H_{e}, t \in H_{e}^{* \infty}$, $f \in E_{e}, \chi \in\left(f H_{e}\right)^{*}$, and $t$ is the equivalence class which contains the "string" $\left\langle\varphi_{f g}(\chi)\right\rangle_{f \leqq g \in E_{e}}$. It is tedious, but not difficult, to show that $\psi$ is well defined and that it is a monomorphism. We can now state our next result.

THEOREM 2.2. Let all notation be as in the preceding paragraph. Then the direct limit $H_{e}^{* \infty}$ of the system of groups

$$
\left[\left\{\left(f H_{e}\right)^{*}\right\}_{f \in E_{e}} ;\left\{\varphi_{f f^{\prime}}\right\}_{f \leqq f^{\prime}}\right]
$$

can be embedded in a natural way into $H_{e}^{*}$. Moreover, each continuous character of $H$ having support $F$ can be extended to a continuous character of $S$ if and only if 
$H_{e}^{* \infty}$ is isomorphic to $H_{e}^{*}$ under the natural embedding and $F$ is induced by some generating idempotent of $S$.

Proof. We omit the details of the proof as they are rather straightforward and are not particularly enlightening. The proof of the last statement of the theorem is a consequence of Theorem 2.1 .

CoRollary 2.3. If $S$ is a compact commutative semigroup, $H$ is a closed subsemigroup of $S$, and $\chi$ is a continuous character of $H$ such that the least element of $E\left(S_{\chi}\right)$ is a generating idempotent of $S$, then $\chi$ has an extension which is a continuous character of $S$.

Proof. Apply Theorem 2.2 to $F=S_{x}$; since $e \in E_{e}, H_{e}^{* \infty} \cong H_{e}^{*}$ under the natural mapping from $H_{e}^{* \infty}$ into $H_{e}^{*}$.

If $A$ and $B$ are nonvoid subsets of a commutative semigroup $S$, then $A / B$ will denote the set $\{x \in S \mid b x \in A$ for some $b \in B\}$.

LEMMA. If $A$ and $B$ are nonvoid subsets of a commutative semigroup $S$, then

1. $A / B$ is open whenever $A$ is open, and

2. $A / B$ is closed whenever $A$ and $B$ are closed and $S$ is compact.

Proof. Trivial.

Corollary 2.4. Assume $S$ is a compact commutative semigroup, that $H$ is a closed subsemigroup of $S$, and that $\chi$ is a continuous character of $H$. In order that $\chi$ admit an extension which is a continuous character of $S$, it is sufficient that $S_{\chi} / S$ be open.

Proof. Observe that if $e_{0}$ is the least element of $E(S)$, then $S_{x} / S=\bigcup\left\{P_{f} \mid e_{0} \leqq f\right\}$. The fact that $S_{x} / S$ is open implies that $e_{0}$ is a generating idempotent of $S$. Corollary 2.4 then follows from Corollary 2.3.

COROLlary 2.5. Assume $S$ is a compact commutative semigroup and that $H$ is a subsemigroup of $S$ which is both open and closed. Then each continuous character of $H$ can be extended to a continuous character of $S$.

Proof. By the lemma above and the assumption that $H$ is open, it follows that $S_{x} / S$ is open for each continuous character $\chi$ of $H$. The corollary now follows from Corollary 2.4 .

3. Extending idempotent characters. In Theorem 2.1, we obtained necessary and sufficient conditions in order that one be able to extend a continuous character $\chi$ of a closed subsemigroup $H$ of a compact commutative semigroup $S$. Essentially the "group part" of the extension problem is taken care of by condition 1 and the "idempotent part" by condition 2. Our feeling about that theorem is that condition 1 is a satisfactory condition, but that condition 2 is little more than a 
translation of the statement that the continuous idempotent character $\chi^{*}$ of $H$ defined by

$$
\begin{aligned}
\chi^{*}(x) & =1, & & x \in S_{x}, \\
& =0, & & x \notin S_{x}
\end{aligned}
$$

admits an extension as a continuous idempotent character of $S$. Thus it is the purpose of this section to study in more detail the problem of extending continuous idempotent characters. One of the main results obtained here shows that if cne is interested in extending continuous idempotent characters defined on closed subsemigroups of compact commutative semigroups $S$, then one may also assume that $S$ is totally disconnected.

It is the author's belief that the following lemma is known, but lacking an explicit reference, we include a proof.

LEMMA 3.1. Assume that $S$ is a compact commutative semigroup and that $H$ is a closed subsemigroup of $S$. Then the union $K$ of the set of all components of $S$ which intersect $H$ is a closed subsemigroup of $S$.

Proof. That $K$ is a subsemigroup follows from the fact that the product of two components is connected.

We show that $K$ is closed. Assume that $\left\{x_{\alpha}\right\}_{\alpha \in A}$ is a net in $K$ converging to $x \in S$. For each $\alpha$, let $C_{\alpha}$ denote the component of $S$ containing $x_{\alpha}$ and let $h_{\alpha} \in C_{\alpha} \cap H$. Choose a subnet $\left\{C_{\beta}\right\}_{\beta \in B}$ of $\left\{C_{\alpha}\right\}_{\alpha \in A}$ such that $\left\{C_{\beta}\right\}$ converges to some $C$ and $\left\{h_{\beta}\right\}$ converges to some $h$ ( $h$ is necessarily in $H$ ). Since $\lim \sup _{\beta} C_{\beta}$ exists and is connected, $h$ and $x$ belong to the same component of $S$ and $x \in K$.

If $H$ is a closed subsemigroup of a compact commutative semigroup $S$, then $K(H)$ will denote the union of the set of all those components $C$ of $S$ such that $C \cap H$ is not void. By Lemma 3.1, $K(H)$ is a closed subsemigroup of $S$. If $H=S_{x}$ for some character $\chi$ defined on a subsemigroup of $S$, then we write $K_{\chi}$ for $K\left(S_{\chi}\right)$.

THEOREM 3.2. Assume $S$ is a compact commutative semigroup and that $\chi$ is a continuous character of some closed subsemigroup $H$ of $S$. In order that $\chi$ admit an extension to a continuous character of $K(H)$, it is necessary and sufficient that

1. whenever $C$ is a component of $S$ such that $0 \in \chi(C \cap H)$, it follows that $\chi(C \cap H)=0$, and

2. $(x, y, a) \in S_{x} \times S_{x} \times K_{x}$ and $x a=y a$ imply $\chi(x)=\chi(y)$.

Proof. That condition 1 is necessary follows trivially, since any extension of $\chi$ would either map all or none of a component $C$ of $S$ to 0 .

That condition 2 is necessary is also trivial in view of Theorem 1.3 and the fact that the restriction to $K_{x}$ of any extension of $\chi$ to $S$ is a unit-character of $K_{x}$.

Conversely, to see that conditions 1 and 2 are sufficient merely observe that $K(H) \backslash K_{x}$ is an open and closed prime ideal of $K(H)$ and proceed as in the proof of Theorem 2.1 .

The proof of the following corollary is immediate. 
COROLlaRY 3.3. If $\chi$ is a continuous idempotent character of a closed subsemigroup $H$ of a compact commutative semigroup $S$, then $\chi$ can be extended to a continuous idempotent character of $K(H)$ if and only if $\chi(C \cap H)=0$ for each component $C$ of $S$ such that $0 \in \chi(C \cap H)$. Moreover, there is at most one such extension to $K(H)$.

Let $S$ denote any compact commutative semigroup. There exists a totally disconnected compact commutative semigroup $D=D_{S}$ and a continuous homomorphism $\varphi=\varphi_{S}: S \rightarrow D_{S}$ such that $\left\{\varphi^{-1}(d) \mid d \in D_{S}\right\}$ is precisely the set of components of $S$ (see Hofmann and Mostert [5]). The semigroup $D_{S}$ will be called the canonical totally disconnected image of $S$ and $\varphi_{S}$ will be called the projection of $S$ onto $D_{S}$.

Note that if $L$ is a subsemigroup of $D_{S}$, then $\varphi^{-1}(L)$ is a subsemigroup of $S$ which is a union of components of $S$. Conversely, any subsemigroup of $S$ in which is a union of components of $S$ is of the form $\varphi^{-1}(L)$ for some subsemigroup $L$ of $D_{S}$. Similar remarks hold for ideals and prime ideals of $S$ and $D_{S}$.

From these observations, it may be shown that each continuous idempotent character $\chi^{*}$ of $D_{S}$ uniquely determines a continuous idempotent character $\varphi^{-1}\left(\chi^{*}\right)$ of $S$, namely the idempotent character of $S$ whose support is $\varphi^{-1}\left(S_{\chi^{*}}\right)$. Conversely, if $\chi$ is a continuous idempotent character of $S$, then $\chi$ uniquely determines a continuous idempotent character $\varphi(\chi)$ of $D_{S}$, namely the idempotent character of $D_{S}$ whose support is $\varphi\left(S_{\chi}\right)$.

Actually the correspondences

$$
\chi \rightarrow \varphi(\chi) \quad \chi^{*} \rightarrow \varphi^{-1}\left(\chi^{*}\right)
$$

are inverse functions between $E\left(S^{\wedge}\right)$ and $E\left(D_{S}\right)$. These functions are easily seen to be isomorphisms. Thus we obtain the following theorem.

THEOREM 3.4. The semigroup $E\left(S^{\wedge}\right)$ of continuous idempotent characters of a compact commutative semigroup $S$ is iseomorphic to the semigroup $E\left(D_{\hat{S}}\right)$ of continuous idempotent characters of the totally disconnected compact commutative semigroup $D_{S}$.

REMARK. Slightly stronger results are available concerning the continuous idempotent characters of closed subsemigroups $H$ of compact commutative semigroups $S$, but they do not seem to shed further light on the matter so they are omitted.

As above, $S$ denotes a compact commutative semigroup with canonical totally disconnected image $D_{S}$ and projection $\varphi_{S}: S \rightarrow D_{S}$. If $T$ is a subsemigroup of $S$ and $T$ is union of components of $S$, then $D_{T}$ can be identified, in a natural way, with $\varphi(T) \subseteq D_{S}$. We shall make the identification without specific mention of the fact. Similarly, if $\chi$ is a continuous idempotent character of $T$, then one may identify it with its image under the iseomorphism between $E\left(T^{\wedge}\right)$ and $E\left(D_{T}^{\wedge}\right)$ described just prior to the statement of Theorem 3.4. It should be pointed out that 
both of these identifications are quite natural; $D_{T}$ is essentially $T$ with its components "collapsed to points" and the element of $E\left(D_{T}^{\widehat{T}}\right)$ corresponding to $\chi \in E\left(T^{\wedge}\right)$ is merely the character of $D_{T}$ whose value at a "point" $x$ of $D_{T}$ is the same as the value of $\chi$ on the component of $T$ which "collapses" to $x$ (recall that $\chi$ is constant on each component of $T$ ).

Our final theorem states that if one is interested in extending continuous idempotent characters of closed subsemigroups of compact commutative semigroups $S$, then one may as well assume that $S$ is also totally disconnected.

THEOREM 3.5. Assume $\chi$ is a continuous idempotent character of a closed subsemigroup $H$ of a compact commutative semigroup $S$. In order that $\chi$ admit an extension which is a continuous idempotent character of $S$ it is necessary and sufficient that $\chi(C \cap H)=0$ for each component $C$ of $S$ such that $0 \in \chi(C \cap H)$ and if $\chi^{*}$ is the unique extension of $\chi$ to $K=K(H)$, then $\chi^{*}$, considered as a continuous idempotent character of $D_{K} \subseteq D_{S}$, can be extended to $D_{S}$.

The proof is straightforward and is omitted.

\section{REFERENCES}

1. C. W. Austin, Duality theorems for some commutative semigroups, Trans. Amer. Math. Soc. 109 (1963), 245-256.

2. R. O. Fulp, On extending semigroup characters, Proc. Edinburgh Math. Soc. (2) 15 (1967), 199-202.

3. E. Hewitt and K. A. Ross, Abstract harmonic analysis. I, Springer-Verlag, Berlin, 1963.

4. Paul Hill, Characters of commutative semigroups, J. Algebra 5 (1967), 16-24.

5. K. H. Hofmann and P. S. Mostert, Elements of compact semigroups, Merrill, Columbus, Ohio, 1966.

6. K. A. Ross, Extending characters on semigroups, Proc. Amer. Math. Soc. 12 (1961), 988-990.

7. S. Schwarz, Characters of bicompact semigroups, Czechoslovak Math. J. 5 (1955), 24-28.

8. - The theory of characters of commutative Hausdorff bicompact semigroups, Czechoslovak Math. J. 6 (1956), 330-364.

UNIVERSITY OF HOUSTON, Houston, TEXAS 